\title{
Effects of different pretreatments on the pore structure of Chinese yam during microwave freeze drying
}

\author{
Hui Wang ${ }^{1}$, Xu Duan ${ }^{2 *}$, Lujie Zhao ${ }^{2}$, Liuliu Duan², Guangyue Ren ${ }^{2,3}$ \\ (1. School of Chemical Engineering and Pharmacy, Henan University of Science and Technology, Luoyang, Henan 471023, China; \\ 2. College of Food and Bioengineering, Henan University of Science and Technology, Luoyang, Henan 471023, China; \\ 3. Henan Cooperative Innovation Center for Grain Storage Safety, Zhengzhou 450001, China)
}

\begin{abstract}
In order to explore the effects of different pretreatments on the pore structure of Chinese yam during Microwave Freeze Drying (MFD), the samples were treated by typical drying pretreatments including osmosis, blanching and ultrasound, and compared with the untreated samples. The results showed that the pretreatment had significant impacts on the porosity of MFD Chinese yam. Ultrasonic pretreatment could obtain the highest open porosity, and the internal pore network structure of the material showed a uniform sponge shape. The shape of pores obtained by blanching pretreatment was the most irregular. The pore network structure obtained by different pretreatments could result in various product texture and rehydration, which implied that pretreatment methods should be carefully selected according to actual requirements. All these pretreatments could improve the open porosity of MFD Chinese yam, which result in a better rehydration capability. Among them, ultrasonic treatment is worth further investigating.
\end{abstract}

Keywords: microwave freeze drying, pretreatment, pore network structure, Chinese yam, porosity DOI: $10.25165 /$ j.ijabe.20201304.5605

Citation: Wang H, Duan X, Zhao L J, Duan L L, Ren G Y. Effects of different pretreatments on the pore structure of Chinese yam during microwave freeze drying. Int J Agric \& Biol Eng, 2020; 13(4): 232-237.

\section{Introduction}

Chinese yam (Dioscorea opposite Thunb) is one of the most important edible and medicinal plants in China. It is widely cultivated in China and is usually consumed as a popular healthy promoting function food. The main active components of yam tuber are flavonol, polysaccharide and steroidal saponins. Among them, the specific active substance is Chinese yam polysaccharide ${ }^{[1]}$. Many studies have confirmed that Chinese yam has excellent health and pharmacological effects in the fields of medicine and health care $^{[2]}$. Currently, various products of Chinese yam are increasingly demanded by consumers. Therefore, more and more dried products of Chinese yam are appearing in the market.

At present, most of the yam chips are produced by frying, puffing, hot air drying and other processes. High temperature leads to low product quality. Among these methods, hot air drying is a commonly used method for drying Chinese yam ${ }^{[3,4]}$. Hot air drying can accurately control the drying temperature to obtain the appropriate core temperature, but the appearance of the sample is generally poor. The material often suffers from discoloration, shrinkage and nutrient loss due to high drying temperature ${ }^{[5,6]}$. In addition, thermal effects during hot air drying lead to degradation of active substances so that the quality loss of

\section{Received date: 2019-12-19 Accepted date: 2020-03-19}

Biographies: Hui Wang, PhD, Associate Professor, research interests: agricultural products processing and safety, Email: wanghui_peony@163.com; Liuliu Duan, Master candidate, research interests: food chemistry, Email: liu786129855@qq.com; Lujie Zhao, Master candidate, research interests: food chemistry, Email: 1223483275@qq.com; Guangyue Ren, PhD, Professor, research interests: food drying technology, Email: guangyueyao@163.com.

*Corresponding author: Xu Duan, PhD, Professor, research interests: agricultural products processing. Food and Biology Engineering College, Henan University of Science \& Technology, Luoyang, Henan 471023, China. Tel: +86-13653872870, Email: duanxu_dx@163.com. products is far beyond acceptable ${ }^{[7]}$. Vacuum fried yam chip may be an important alternative with lower cost, better texture and flavor, but the oil content of the product cannot be controlled at a very low level ${ }^{[8]}$. Compared with vacuum frying, freeze drying (FD) can process Chinese yam at lower temperature. The FD products of Chinese yam have higher nutritional value and lower quality loss, which can meet the requirements of consumers ${ }^{[9]}$. But FD has high energy consumption, expensive operation and long drying time. Its application in industry is limited by these shortcomings ${ }^{[10]}$.

In recent years, Microwave Freeze Drying (MFD) has attracted wide attention for its ability to produce high-quality products. Compared with FD, MFD can reduce energy consumption by about $30 \%{ }^{[11]}$. Qian et al. ${ }^{[12]}$ showed that the combination of freeze drying and microwave drying could significantly reduce the energy consumption of freeze drying carrot. The results also showed that retention of carotene and rehydration rates were similar to those of freeze-dried products, meanwhile, the drying time was greatly shortened. Zheng et al. ${ }^{[13]}$ reported that the product quality of MFD was almost the same as that of FD. Therefore, MFD has the potential to replace the traditional freeze drying.

It is well known that FD products have porous structure, which leads to its products with unique taste and rehydration property. The porosity is an important physical property characterizing the texture and the quality of dry and intermediate moisture foods. In recent studies, it has been recognized that the porous structure plays an important role in determining the quality of food. Many problems in food research have been found to be rooted in events that occur at the microstructure level (below $100 \mu \mathrm{m}$ ), where the extent of physicochemical and biochemical changes in postharvest tissues is significantly dependent on cellular organization ${ }^{[14]}$. It appears that food structure is closely associated to product performance (chemical and biological stability, textural properties, transport properties, etc.) and that structure in turn depends on 
processing history. In addition, porosity is an important parameter in the prediction of the diffusional properties of cellular foods. One of the main affected transport properties is the diffusivity of gases and liquids in the material. The diffusivity in the gaseous phase in a porous solid is proportional to the porosity of the material and irreversibly proportional of the tortuosity ${ }^{[15]}$. The porosity of the material expresses the ratio of open (empty) space of the material to that of total material volume. Huizenga and $\mathrm{Smith}^{[16]}$ found that the effective vapor diffusivity in porous solids increased with increasing porosity and average pore size. Karathanos and Saravacos ${ }^{[17]}$ found similar results for starch materials. At present, there are few reports on the behavior of pore changes in dried products during FD. Moreover, pore formation is more complex in MFD process ${ }^{[18]}$. Therefore, the formation of porous structure in MFD process needs to be further studied.

Apart from the drying process, different pretreatments also could affect the pore formation during MFD. At present, wildly used pretreatment methods of drying include blanching, osmotic, ultrasonic treatment, etc ${ }^{[19]}$. Blanching is the most commonly used thermal pretreatments before processing of agricultural products as it can destroy enzymes, which cause deterioration reactions, off-flavor and undesirable changes in color, texture and nutrients. Osmotic treatment has received much attention due to its low cost and multiple functions. Li et al. ${ }^{[20]}$ studied the effect of pectin osmosis on the texture properties of dehydrated apple cube, and they found that although the change of pectin content did not significantly affect the volume expansion ratio of dried apple cube, it still could affect the internal pore diameter and pore wall thickness. Ultrasound technology has been directly or indirectly used as a pretreatment in many drying and/or dehydration applications. Ultrasonic pretreatment can be used before drying and osmotic dehydration of vegetables and fruits. Studies showed that effective water diffusivity increases after the application of ultrasound causing a reduction of about $16 \%$ in the drying time ${ }^{[21]}$. At the same time, different pretreatments will also affect the pore structure of dried materials. However, the effect of typical pretreatments on pore formation of Chinese yam during MFD process has not been reported.

The investigation for the effect of different pretreatments on pore structure of MFD Chinese yam can help to improve the product quality, which is a crucial link in the industrialization of microwave freeze drying. Therefore, the object of this study is to compare the effect of different pretreatments on the pore characteristics of Chinese yam during MFD process, and investigate the porous structure change law of MFD Chinese yam.

\section{Materials and methods}

\subsection{Materials and drying with different pretreatments}

Fresh Chinese yam (Dioscorea opposite Thunb) was purchased from a local market in Luoyang, China. The Chinese yams were washed, peeled and sliced into $4 \mathrm{~mm}$ thick segments. The samples were round and had a diameter of approximately $20 \mathrm{~mm}$. In order to study the effects of different pretreatments on the pore characteristics of Chinese yam during MFD, fresh Chinese yam slices were treated by the following three methods. It should be noted that different pretreatments have complex technical processes, which could lead to various structures. In order to simplify the comparison results of various pretreatments, only common treatment parameters were used without considering optimal conditions.
Osmotic pretreatment ${ }^{[22]}$ : the samples were immersed in $5 \%$ salt solution with a solid-liquid ratio of $1: 8$ for $3 \mathrm{~h}$ at $30^{\circ} \mathrm{C}$ in an electrothermal constant temperature water bath (HH-S4, Beijing Kewei Yongxing Instrument Co., Ltd.). After immersion, the salt solution adhering to the sample surface was washed with deionized water, and the water on the surface was absorbed by filter paper.

Blanching pretreatment ${ }^{[23]}$ : the samples were blanched in hot water at $85^{\circ} \mathrm{C}$ for $1 \mathrm{~min}$ in an electrothermal constant temperature water bath (HH-S4, Beijing Kewei Yongxing Instrument Co., Ltd.) and the water on the surface of the material was absorbed by filter paper.

Ultrasound pretreatment: the ultrasound pretreatment system was developed by the authors ${ }^{[24]}$. Its main components are an ultrasonic generator and an ultrasonic transducer. Power ultrasound was delivered in the tank at a fixed frequency of $25 \mathrm{kHz}$ under the power of $300 \mathrm{~W}$. Samples were immersed in the water in the tank at $30^{\circ} \mathrm{C}$ for $30 \mathrm{~min}$, and then the water on the surface of the material was absorbed by filter paper.

After various pretreatments, the samples $(1 \mathrm{~kg})$ were placed in a polypropylene tray and frozen at $-25^{\circ} \mathrm{C}$ for at least $8 \mathrm{~h}$, and then microwave freeze drying was carried out. The microwave freeze dryer was developed by authors. As shown in Figure 1, an independent polypropylene drying cavity was set up in a rectangular resonant cavity, which could effectively avoid the coronal discharge under the vacuum condition. The pressure of the drying cavity was operated within a range of $10 \mathrm{kPa}$ to $30 \mathrm{kPa}$ (absolute pressure). The power of the microwave could be adjusted continually. The core temperatures of the materials were detected by an optic fiber sensor. In the experiment, a fixed microwave power density loading levels $(0.225 \mathrm{~W} / \mathrm{g})$ were applied. All MFD processes were carried out at a cavity pressure of $100 \mathrm{~Pa}$ and a cold trap temperature of $-40^{\circ} \mathrm{C}$. All of the experiments above were repeated three times and the average of the results was used for the analysis.

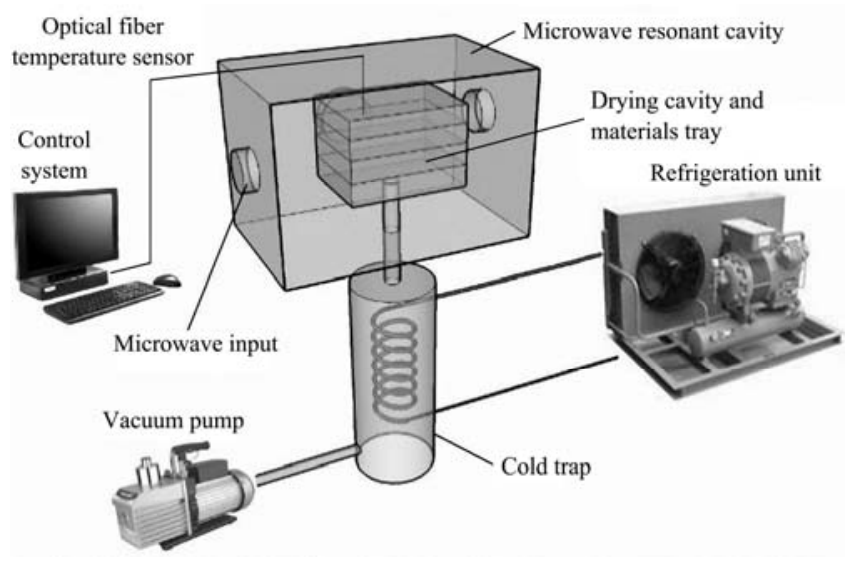

Figure 1 Schematic diagram of microwave freeze dryer

\subsection{Detection methods}

\subsubsection{Determination of total porosity $(\varepsilon)$}

The porosity of the sample was determined by the pycnometer. At 30 min intervals during the drying processes samples were taken out, crushed it to below $0.15 \mathrm{~mm}$, soaked in $20^{\circ} \mathrm{C}$ n-hexane, and then held on a pycnometer for $30 \mathrm{~min}$. The weighing was performed on a digital balance. The tests were performed in triplicate. The porosity of samples under different drying conditions was calculated according to the Equations (1) and (2) as follows ${ }^{[25]}$.

$$
\rho_{s}=\frac{m_{s} \rho}{m_{s}+m_{1}-m_{2}}
$$




$$
\varepsilon=\left(1-\frac{m_{s}}{V \rho_{s}}\right)
$$

where, $m_{s}$ is the weight of samples, $\mathrm{g}$; $\rho$ is the density of $\mathrm{n}$-hexane at $20^{\circ} \mathrm{C}, \mathrm{g} / \mathrm{cm}^{3} ; m_{1}$ is the weight of the pycnometer filled with $\mathrm{n}$-hexane, $\mathrm{g} ; m_{2}$ is the mass of the pycnometer filled with samples and n-hexane, g; $V$ is the volume of samples, $\mathrm{cm}^{3} ; \rho_{s}$ is the true density of the samples, $\mathrm{g} / \mathrm{cm}^{3}$.

\subsubsection{Measurement of opening porosity $\left(\varepsilon_{0}\right)$}

The pores that are interconnected within the material or connected to the surface of the material are called open pores, and the pores that are divided into independent pores inside the material are called closed pores. The sample was placed in a $50 \mathrm{~mL}$ beaker and immersed by n-hexane. After two hours, the saturated sample was taken out, wiped out the free n-hexane on the surface of the sample, and then weighed with an electronic balance. Each experiment was performed in triplicate. The open porosity was calculated according to Equation (3) ${ }^{[25]}$.

$$
\varepsilon_{0}=\frac{m_{2}-m_{1}}{\rho V}
$$

where, $m_{1}$ is the weight of the sample before immersion, $\mathrm{g} ; m_{2}$ is the weight of the immersed sample, $\mathrm{g} ; \rho$ is the density of n-hexane, $\mathrm{g} / \mathrm{cm}^{3} ; V$ is the sample volume, $\mathrm{cm}^{3}$.

\subsubsection{Measurement of closed pore porosity $\left(\varepsilon_{1}\right)$}

According to Equation (4), the closed porosity is calculated as follows ${ }^{[25]}$ :

$$
\varepsilon_{1}=\varepsilon-\varepsilon_{0}
$$

where, $\varepsilon$ is the total porosity of the sample, $\%$; $\varepsilon_{0}$ is the open porosity, $\%$.

\subsubsection{Scanning electron microscope analysis}

The microwave freeze dried sample was placed on conductive adhesive tabs, mounted on a scanning electron microscope (S4800, Hitachi, Japan) tubs and coated with gold-palladium in a sputter coater. The internal pore morphology of the coated samples was observed at an accelerating voltage of $4 \mathrm{kV}$.

\subsubsection{Pore size and pore distribution}

The microwave freeze dried sample was analyzed by a Mercury Porosimetry (AutoPore IV 9500, Micromeritics instrument corporation, USA). The AutoPore IV 9500 provided full-scale resolution in the following pressure ranges: low pressure (4-345 $\mathrm{kPa}$ ), and high pressure (0.1-414 MPa). For testing, the sample was placed in the dilatometer, and then cleansed of absorbed and/or adsorbed gases by degassing in a vacuum $(4 \mathrm{kPa})$. The dilatometer, still under vacuum, was then filled with mercury. The pressure in the dilatometer was built up gradually to a final pressure of $414 \mathrm{MPa}$ for the high-pressure tests and $345 \mathrm{kPa}$ for low-pressure tests, in approximately $15 \mathrm{~min}$. The mean pore diameter was given from the AutoPore software.

\section{Results and analysis}

\subsection{Effects of different pretreatments on total porosity of} Chinese yam during microwave freeze drying

As shown in Figure 2, it was found that there was a significant difference in porosity variation between untreated samples and pretreated samples, which implied that pretreatment could significantly affect the total porosity of MFD Chinese yam. According to Figure 2, the porosity of the untreated sample showed a down trend in the drying time of 0-60 min. The possible reason was that the volume shrinkage caused by a large amount of free water sublimation affected the formation of pores. In the period of 0-30 min, the porosity of pretreated samples increased, and from
$30 \mathrm{~min}$ to $60 \mathrm{~min}$, the porosity of pretreated samples dropped again. This could be due to the fact that pretreatment destroyed the internal structure of the samples to a certain extent, resulting in a certain micro-pore structure, which reduced the water diffusion resistance, and decreased the volume shrinkage phenomenon. This was conducive to the formation of pores during this drying stage. Therefore, the total porosity of the pretreated samples showed an upward trend in the period of 0-30 min. The total porosity of ultrasonic pretreated samples was larger than that of osmotic and blanching pretreatments in the drying time of 0-30 min. This indicated that ultrasonic pretreatment had a relatively higher impact on the material, leading to more pores formation. Then, the total porosity of various pretreated samples decreased in varying degrees in drying time of 30-60 min. The possible reason was that the volume shrinkage of samples increased and destroyed some formed pores. In addition, the porosity of ultrasonic pretreated samples had the fastest fall trend, which implied the samples treated by ultrasound were easier to be compressed, resulting in a decrease in total porosity. After drying time of $60 \mathrm{~min}$, the total porosity of various pretreated samples kept similar trends as that of the untreated samples, and showed a trend of going up first and then down. Compared with untreated samples, the total porosity of the pretreated samples was higher at the beginning stage, and became lower at the end of drying. The reason was that pretreatments destroyed the cell wall structure resulting in fast ice sublimation at the early drying stage. As a result, more pores could form at this stage. Pretreatments improved the heat and mass transfer efficiency and water diffusion rate in the early stage of drying, and then accelerated the formation of pores. On the other hand, pretreatments reduced the effect of volume shrinkage on pore formation. As a result, the total porosity of pretreated samples at the early stage of drying was higher. The order of total porosity from high to low was ultrasonic, blanching and osmosis pretreated samples respectively. This also implied that ultrasound could accelerate the water diffusion rate, resulting in more pores formation $^{[26]}$. At the end stage of drying, long-term microwave heating could lead to local high temperature of the sample, resulting in some ice melting, and then local high moisture content and high temperature could lead to the collapse of the matrix, so the porosity showed a downward trend. When the drying completed, the order of total porosity from high to low was untreated, osmosis, blanching and ultrasonic pretreatment. This indicated that collapse was more likely to occur at the end of drying, and the collapse phenomenon reduced the total porosity.

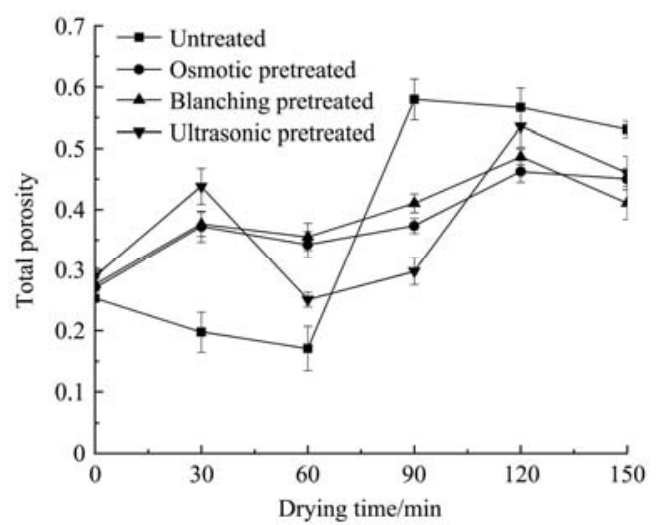

Figure 2 Variation of total porosity of Chinese yam under different pretreatments during MFD process

In order to further investigate the pore form evaluation under various pretreatments during MFD process, the changes of opening 
and closed pores were detected, which were shown in Figures 3 and 4. As shown in Figure 3, the opening porosity of different samples increased with the drying proceeding. After $60 \mathrm{~min}$, the difference in opening porosity between pretreated and untreated samples increased significantly. When the drying completed, the samples undergoing blanching and ultrasonic pretreatments had the highest opening porosity. The opening porosity of the samples pretreated by osmosis, blanching and ultrasound were about 1.23 , 1.98 and 1.92 times of that of untreated samples respectively. The results showed that pretreatments could effectively improve the proportion of opening pores. Moreover, it was illustrated that the samples pretreated by ultrasound had the largest proportion of opening pores, which made them have a better rehydration ${ }^{[20]}$. Therefore, ultrasound is a recommendable treatment method for MFD Chinese yam.

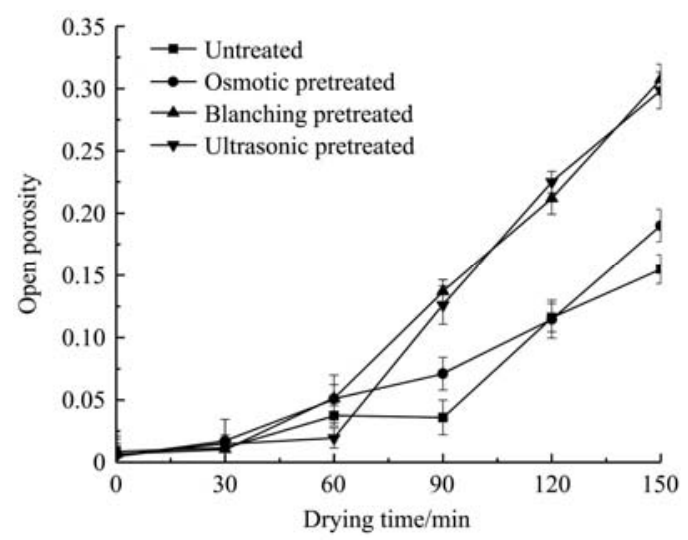

Figure 3 Variation of open porosity of Chinese yam under different pretreatments during MFD process

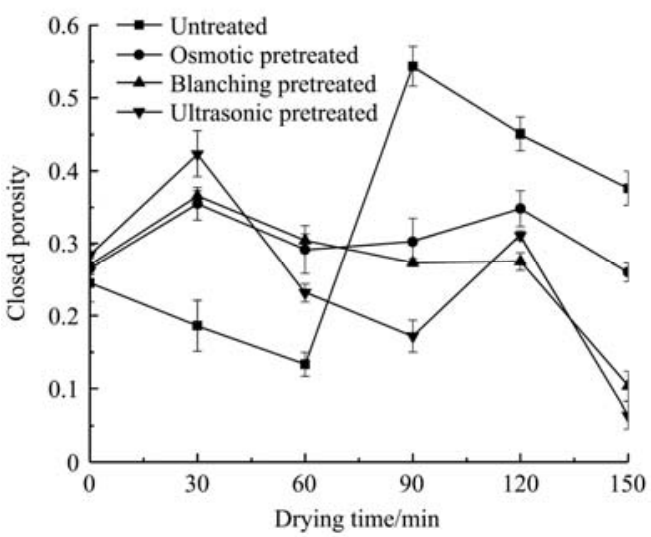

Figure 4 Variation of closed porosity of Chinese yam under different pretreatments during MFD process

As shown in Figure 4, the changing trend of closed porosity was similar to that of total porosity. The untreated samples exhibited a trend of downward-upward-downward. The pretreated samples showed a trend of upward-downward-upward-downward during the whole drying process. Within drying time of 0-30 $\mathrm{min}$, the pretreated and untreated samples showed the opposite tendency. For pretreated samples, faster water diffusion and sublimation led to some pore formation, and compressive stress caused by shrinkage also was low in the early drying stage. Therefore, the closed porosity of pretreated samples showed an upward trend during this period. During the period of 30-60 min, the shrinkage stress inside the material became higher and the closed pores were compressed. Therefore, the closed pore porosity showed a downward trend.
The closed porosity of osmotic samples increased gradually after $60 \mathrm{~min}$, and the closed porosity of ultrasonic and blanching treated samples gradually recovered after $90 \mathrm{~min}$. At the end of drying, the closed porosity of all pretreated samples showed a downward trend. Compared with Figures 2 and 3, it could be concluded that lots of closed pores constantly transformed into open pores during the drying process. In addition, some network structures collapsed because of local hyperthermia under action of microwave energy, leading to the transformation of closed pore into open pore. From Figure 4, it could be concluded that the pore structure of the pretreated samples was looser and collapse was more likely to occur than that of the untreated samples. The change of pore structure will affect the texture characteristics of dried samples ${ }^{[27]}$.

\subsection{Effects of different pretreatments on the pore structure of MFD Chinese yam}

In order to further explore the effect of different pretreatments on the pore structure evolution of Chinese yam during microwave freeze drying process, the variation of total porosity, open porosity and closed porosity under different moisture content were compared as shown in Figure 5. The results showed that with the decrease in water content, only the open porosity increased continuously among the three kinds of porosity. As drying proceeding, when the water content of untreated samples ranged from about $40 \%$ to $10 \%$, the closed porosity decreased rapidly, and the open porosity increased rapidly. This could be due to the matrix collapses during this period, meanwhile, some closed pores transformed into open pores. The moisture content of the samples treated by osmosis, blanching and ultrasound was about 15\%, 16\% and $22 \%$, respectively when a large number of closed pores were transformed into open pores. For blanching and ultrasonic pretreated samples, when drying was finished, the open porosity was 2.97 and 4.97 times of the closed porosity, respectively. This meant that blanching and ultrasonic pretreatment were easier to obtain porous network structure and products with higher rehydration. The opening porosity of the osmosis treated samples was only 0.73 times of the closed porosity, but it was still 1.41 times of that of the untreated samples. This result showed that all three pretreatments could increase the opening porosity of the material.

Scanning electron microscopy was used to observe the pore morphology of different pretreated Chinese yam when the MFD process was finished. As was shown in Figure 6, it was illustrated that the untreated samples had fewer and smaller pores, less pore network structure, relatively uniform cell structure and less cell damage. Pretreatments could increase the pore size of samples, and also could lead to damage to the cell structure. The porous network structure of ultrasound treated samples was the loosest, and showed uniform sponge shape, resulting in soft texture. The reason was that the ultrasonic wave could produce lots of uniform micro-channels in the material due to the mechanical stresses associated with wave transmission ${ }^{[28]}$. The diffusion of water during microwave freeze drying resulted in the expansion of these micro-channels and the uniform spongy network structure was produced. This result agreed with other investigations about ultrasound treatment for drying products ${ }^{[29]}$. The porous network structure of blanching treated samples was tighter than that of ultrasound treated samples, and the pore shape was very irregular. The osmotic pretreatment could lead to cells shrinkage under osmotic pressure, and the cells shrank tightly to form lots of closed pores. 


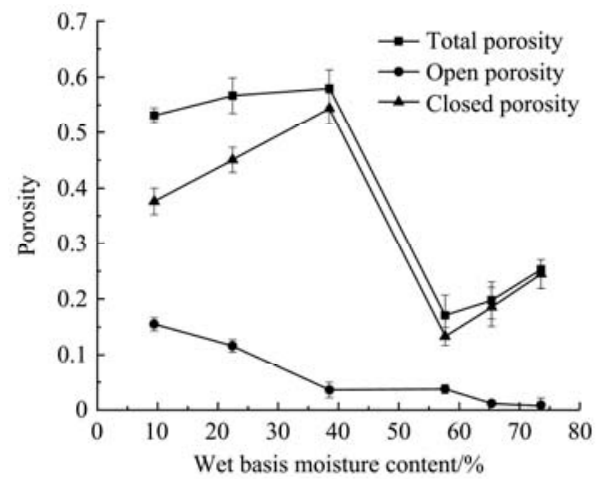

a. Untreated

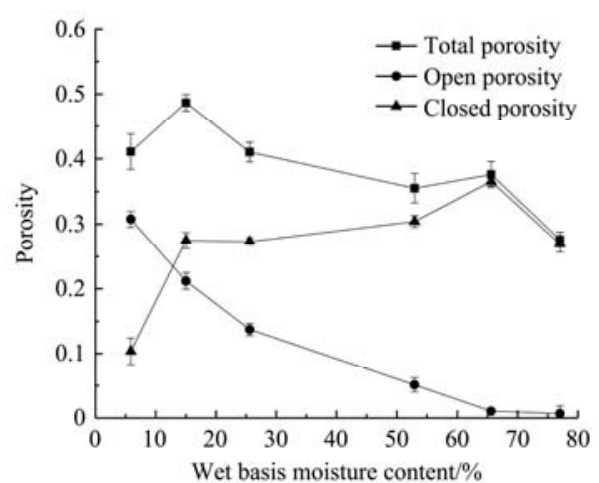

c. Blanching

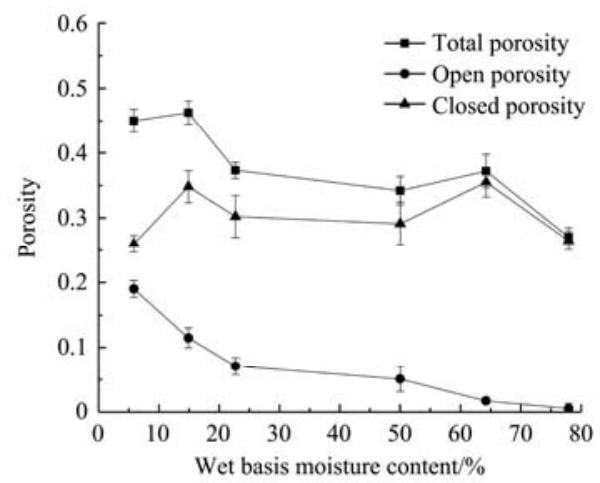

b. Osmosis

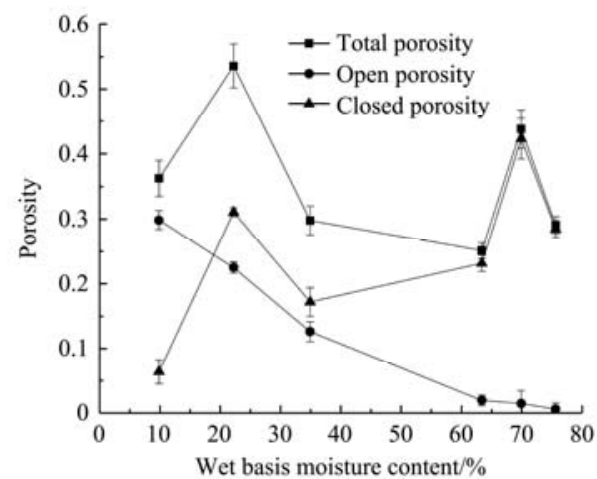

d. Ultrasound

Figure 5 Porosity of MFD Chinese yam under different moisture content

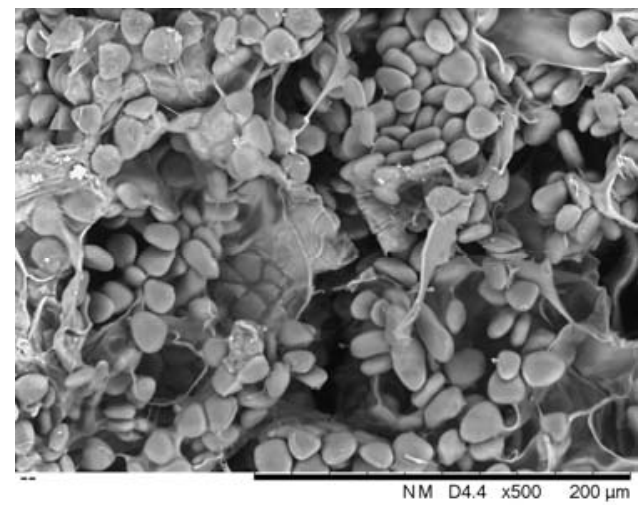

a. Untreated

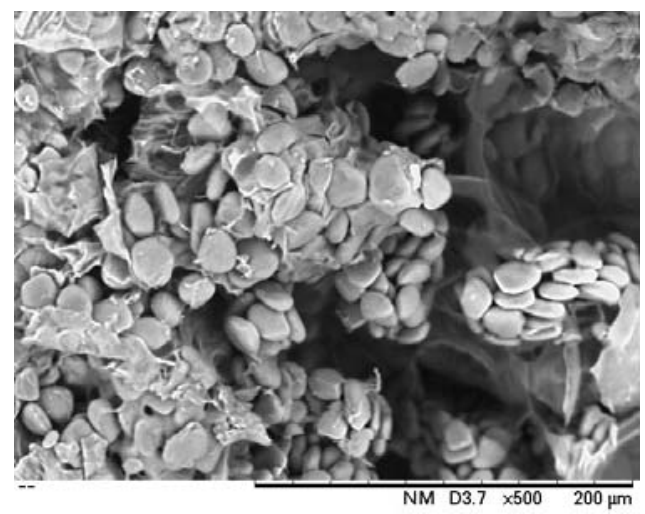

c. Blanching

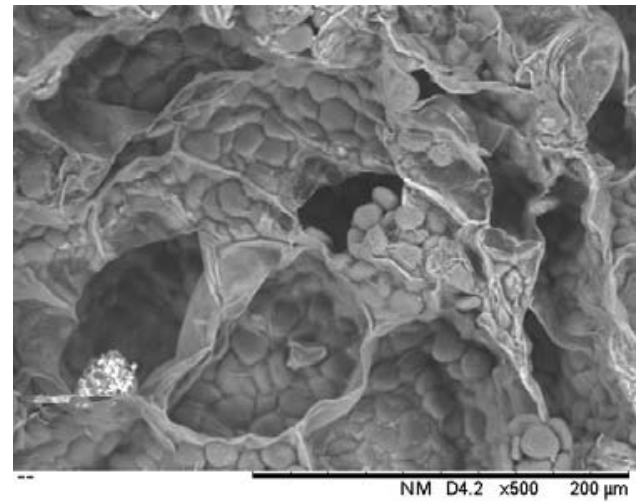

b. Osmosis

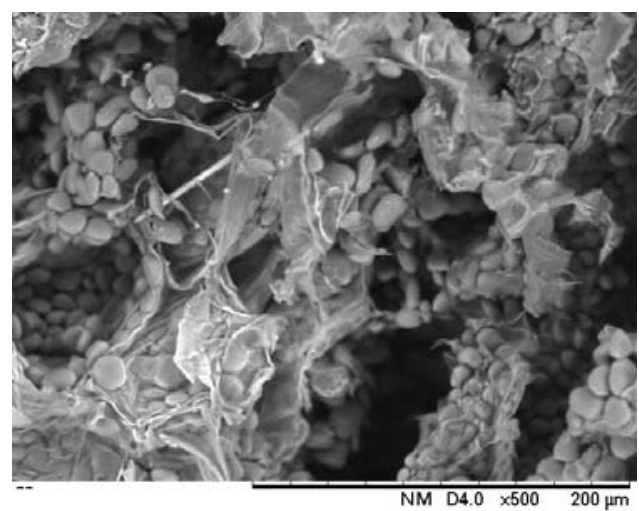

d. Ultrasound

Figure 6 SEM images of Chinese yam undergoing different pretreatments $(\times 500)$

Figure 7 showed the pore size distribution of dried Chinese yam under different pretreatments. The peak of Figure 7 represented the amount of mercury intrusion, and the amount of mercury intrusion represented the number of pores. It was found that the mercury intrusion was in the descending order of ultrasound, blanching, untreated, osmosis when the pore size ranged from $10 \mathrm{~nm}$ to $10^{5} \mathrm{~nm}$. When the pore size was above $10^{5} \mathrm{~nm}$, the mercury intrusion was in the descending order of osmosis, ultrasound, blanching, untreated. This implied that ultrasonic pretreatment could obtain more small size pores and osmotic pretreatment could obtain more large size pores. The possible reason was that ultrasonic pretreatment resulted in many 
micro-channels where ice crystals sublimated, which produced small size pores rather than destroyed the cell structure. However, osmotic pretreatment made the cell structure shrink under osmotic pressure. When the cell structure was stretched, the pore size became larger.

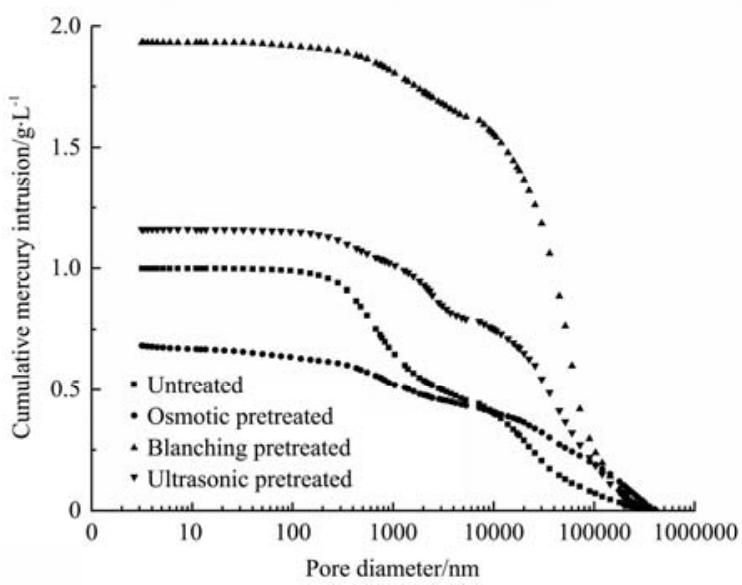

Figure 7 Mercury test results of MFD Chinese yam undergoing different pretreatments

\section{Conclusions}

Typical pretreatments for MFD Chinese yam, including ultrasound, blanching and osmosis, have significant effects on the product pore structure. The open porosity of the dried products is in the descending order of ultrasound, blanching, osmosis and untreated samples. Among all pretreatments, the highest open porosity can be obtained by ultrasonic pretreatment, and the pore network structure of ultrasonically treated samples exhibits a uniform spongy shape. In addition, the pore shape obtained by blanching is the most irregular. As a result, all these pretreatments could improve the open porosity of MFD Chinese yam, which result in a better rehydration capability. Among them, ultrasonic treatment is worth further investigating.

\section{Acknowledgements}

The authors acknowledge that this work was financially supported by the National Natural Science Foundation of China (Contract No. 31671907 and No. 31972207).

\section{[References]}

[1] Li Z Q, Cao W F. Advances in pharmacological effects of yam and its main active ingredients. Chinese Journal of Gerontology, 2013; 33(8): 1975-1976. (in Chinese)

[2] Zhou Y, Guo H, Zhou J. Study on main nutrients in iron Dioscorea opposita Thunb. Food and Nutrition in China, 2011; 17(3): 69-71. (in Chinese)

[3] Xiao H W, Yao X D, Lin H, Yang W X, Meng J S, Gao Z J. Effect of SSB (superheated steam blanching) time and drying temperature on hot air impingement drying kinetics and quality attributes of yam slices. Journal of Food Process Engineering, 2012; 35(3): 370-390.

[4] Ju H Y, Elmashad H M, Fang X M, Pan Z L, Xiao H W, Liu Y H, et al. Drying characteristics and modeling of yam slices under different relative humidity conditions. Drying Technology, 2016; 34(3): 296-306.

[5] Rajkumar G, Shanmugam S, Galvâo M D, Neta M T, Sandes R D, Mujumdar A S, et al. Comparative evaluation of physical properties and aroma profile of carrot slices subjected to hot air and freeze drying. Drying Technology, 2017; 35(6): 699-708.

[6] Ju H Y, Law C L, Fang X M, Xiao H W, Liu Y H, Gao, Z J. Drying kinetics and evolution of the sample's core temperature and moisture distribution of yam slices (Dioscorea alata $\mathrm{L}$.) during convective hot-air drying. Drying Technology, 2016; 34(11): 1297-1306.
[7] Qian Z, Zhang G C, Mu G, Liu Y. Freeze and microwave vacuum combination drying technique for sea cucumber. International Journal of Agricultural and Biological Engineering, 2012; 5(3): 83-89.

[8] Oginni O C, Sobukola O P, Henshaw F O, Afolabi W A, Munoz L A. Effect of starch gelatinization and vacuum frying conditions on structure development and associated quality attributes of cassava-gluten based snack. Food Structure, 2015; 3: 12-20.

[9] Zhang Z D, Gao W Y, Li X, Jiang Q Q, Xia Y Z, Wang H Y, et al. Effect of different drying methods on the physicochemical and functional properties of Dioscorea opposita Thunb. starch. Starch - Stärke, 2013; 65(3-4):8. doi: 10.1007/s11130-019-00729-7.

[10] Duan X, Yang X T, Ren G Y, Pang Y Q, Liu L L, Liu Y H. Technical aspects in freeze-drying of foods. Drying Technology, 2016; 34(11): 1271-1285.

[11] Duan X, Liu W, Ren G.Y, Liu W C, Liu Y H. Comparative study on the effects and efficiencies of three sublimation drying methods for mushrooms. Inter J Agric \& Biol Eng, 2015; 8(1): 91-97.

[12] Qian G L, Zhang Q, Cui Z W. Reducing energy consumption of carrot slices dehydration by combined vacuum microwave and freeze drying. Trans of the CSAE, 2011; 27(6): 387-392. (in Chinese)

[13] Cui Z W, Li C Y, Song C F, Song Y. Combined microwave-vacuum and freeze drying of carrot and apple chips. Drying Technology, 2008; 26(12) 1517-1523.

[14] Ong S P, Law C L. Microstructure and optical properties of salak fruit under different drying and pretreatment conditions. Drying Technology, 2011; 29(16): 1954-1962.

[15] Satterfield C N, Sherwood T K. The role of diffusion in catalysis. Addison-Wesley Pub. Co., 1963; 118p.

[16] Huizenga D G, Smith D M. Knudesen diffusion in random assemblages of uniform spheres. AIChE Journal, 1986; 32(1): 1-6.

[17] Karathanos V T, Saravacos G D. Porosity and pore size distribution of starch materials. Journal of Food Engineering, 1993; 18(3): 259-280.

[18] Duan L L, Duan X, Ren G Y. Structural characteristics and texture during the microwave freeze drying process of Chinese yam chips. Drying Technology, In Press, doi: 10.1080/07373937.2019.1600142.

[19] Deng L Z, Mujumdar A S, Zhang Q, Yang X H, Wang J, Zheng Z A, et al Chemical and physical pretreatments of fruits and vegetables: Effects on drying characteristics and quality attributes-a comprehensive review. Critical Reviews in Food Science and Nutrition, 2019; 59(9): 1408-1432.

[20] Li X, Bi J F, Jin X, Li X, Zhao Y Y, Song Y. Effect of pectin osmosis or degradation on the water migration and texture properties of apple cube dried by instant controlled pressure drop drying (DIC). LWT, 2020; 109202.

[21] Fernandes F A N, Rodrigues S. Dehydration of sapota (Achras sapota L.) using ultrasound as pretreatment. Drying Technology, 2008; 26(10): 1232-1237.

[22] Li L L, Zhang M, Wang W Q. Ultrasound-assisted osmotic dehydration pretreatment before pulsed fluidized bed microwave freeze-drying (PFBMFD) of Chinese yam. Food Bioscience, 2020; 35: 100548. doi: 10.1016/j.fbio.2020.100548.

[23] Li L L, Zhang M, Zhou L Q. A promising pulse-spouted microwave freeze drying method used for Chinese yam cubes dehydration: Quality, energy consumption, and uniformity. Drying Technology, 2019; 1-14.

[24] Duan X, Zhang M, Li X L, Mujumdar A S. Ultrasonically enhanced osmotic pretreatment of sea cucumber prior to microwave freeze drying. Drying Technology, 2008; 26(4): 420-426.

[25] Duan L L, Duan X, Ren G Y. Evolution of pore structure during microwave freeze-drying of Chinese yam. Inter J Agric \& Biol Eng, 2018 11(6): 208-212.

[26] Musielak G, Mierzwa D, Kroehnke J. Food drying enhancement by ultrasound-A review. Trends in Food Science \& Technology, 2016; 56: $126-141$.

[27] Porciuncula B D A, Segura L A, Laurindo J B. Processes for controlling the structure and texture of dehydrated banana. Drying Technology, 2016 34(2): 167-176.

[28] La Fuente C I A, Tadini C C. Unripe banana flour produced by air-drying assisted with ultrasound-description of the mechanisms involved to enhance the mass transfer in two approaches. International Journal of Food Engineering, 2017; 13(11): 13-19.

[29] Amami E, Khezami W, Mezrigui S, Badwaik L S, Bejar A K, Perez C T, Kechaou N. Effect of ultrasound-assisted osmotic dehydration pretreatment on the convective drying of strawberry. Ultrasonics Sonochemistry, 2017; 36: 286-300. 